\title{
Left Baire-1 Compositors and Continuous Functions
}

\author{
Jonald P. Fenecios ${ }^{1}$ and Emmanuel A. Cabral ${ }^{2}$ \\ ${ }^{1}$ Department of Mathematics, Ateneo de Davao University, E. Jacinto Street, 8000 Davao City, Philippines \\ ${ }^{2}$ Department of Mathematics, Ateneo de Manila University, Loyola Heights, 1108 Quezon City, Philippines
}

Correspondence should be addressed to Jonald P. Fenecios; jpfenecios@addu.edu.ph

Received 22 February 2013; Revised 23 September 2013; Accepted 26 September 2013

Academic Editor: Hernando Quevedo

Copyright (C) 2013 J. P. Fenecios and E. A. Cabral. This is an open access article distributed under the Creative Commons Attribution License, which permits unrestricted use, distribution, and reproduction in any medium, provided the original work is properly cited.

\begin{abstract}
We showed that the class of left Baire-1 compositors is precisely the class of continuous functions. This answers a characterization problem posed in the work by Zhao (2007, page 550) and settles in the affirmative the conjecture in the work by Fenecios and Cabral (2012, page 43). Moreover, based on the above result we provide a new proof that the class of functions $\mathscr{B}_{\delta, \mathscr{T}}$ where $\mathcal{\delta}$ is the class of all positive continuous functions and $\mathscr{T}$ is the class of all positive constants as defined in the work by Bakowska and Pawlak (2010) is exactly the class of all continuous functions.
\end{abstract}

\section{Introduction}

Let $\left(X, d_{X}\right)$ be a complete separable metric space. In the classical sense a function $f: X \rightarrow \mathbb{R}$ where $\mathbb{R}$ is the real line is Baire-1 if, for every open set $G \subset \mathbb{R}, f^{-1}(G)$ is an $F_{\sigma}$ set in $X$. Equivalently, a function $f: X \rightarrow \mathbb{R}$ is Baire-1 if, for every closed set $K$ in $X$, the restriction $\left.f\right|_{K}$ has a point of continuity in $K$. In fact, the set of points of continuity of $f$ is a residual subset of $X$.

Recently, Lee et al. [1] discovered a new equivalent definition of Baire- 1 functions. A function $f: X \rightarrow \mathbb{R}$ is Baire-1 if and only if for each $\epsilon>0$ there is a positive function $\delta: X \rightarrow \mathbb{R}^{+}$such that for any $x, y \in X$

$$
d_{X}(x, y)<\min \{\delta(x), \delta(y)\} \Longrightarrow|f(x)-f(y)|<\epsilon .
$$

Various investigations have been done on the class of Baire-1 functions as well as on its subclasses using the $\epsilon-\delta$ characterization. See, for instance, [2-4].

Let us recall in [4] that the notion of the left Baire-1 compositors was introduced as a natural counterpart of the notion of right Baire- 1 compositors. A function $f: \mathbb{R} \rightarrow \mathbb{R}$ is a left Baire-1 compositor if for every Baire-1 function $g: X \rightarrow$ $\mathbb{R}$ the composition of functions $f \circ g: X \rightarrow \mathbb{R}$ is Baire1. Unlike the right Baire-1 compositors there is no known characterization for the left Baire-1 functions. It was shown in [3] that there exists a function with finite set of discontinuity which is not a left Baire-1 compositor. This observation leads to the conjecture that left Baire-1 functions must have very nice properties. We proved that this is indeed the case: the class of the left Baire-1 compositors is precisely the class of all continuous functions defined on $\mathbb{R}$. Moreover, we use this fact to reestablish that $\mathscr{B}_{\mathscr{C}^{+}, \mathcal{T}} \subset \mathscr{C}$ where $\mathscr{C}^{+}$is the class of all positive continuous functions, $\mathscr{T}$ is the class of all positive real constants, and $\mathscr{C}$ is the class of all continuous functions on $\mathbb{R}$ as defined in [2].

\section{Main Result}

Let us denote the $\min \{a, b\}$ by $a \wedge b$. For easy reference, we present the following useful results.

In $[4$, Proposition 1], it was shown that $f: \mathbb{R} \rightarrow \mathbb{R}$ is Baire-1 if and only if for every positive continuous function $\epsilon$ there is a corresponding positive function $\delta$ such that for any $x, y \in \mathbb{R}$

$$
\begin{aligned}
|x-y|<\delta(x) \wedge \delta(y) \\
\quad \Longrightarrow|f(x)-f(y)|<\epsilon(f(x)) \wedge \epsilon(f(y)) .
\end{aligned}
$$


On the other hand, following [2] a function $f: \mathbb{R} \rightarrow \mathbb{R}$ belongs to $\mathscr{B}_{\mathcal{S}, \mathscr{T}}$ if for every $\epsilon \in \mathscr{T}$ there exists a function $\delta \in \mathcal{S}$ such that for any $x, y \in \mathbb{R}$

$$
\begin{aligned}
|x-y|<\delta & (x) \wedge \delta(y) \\
& \Longrightarrow|f(x)-f(y)|<\epsilon(f(x)) \wedge \epsilon(f(y)),
\end{aligned}
$$

where $\mathcal{S}$ and $\mathscr{T}$ are families of positive functions defined on $\mathbb{R}$.

The following theorem was proved in [2].

Theorem 1. The following holds:

$$
\mathscr{B}_{l s c^{+}, \text {Const }^{+}} \subset \mathscr{C} \text {, }
$$

where $l s c^{+}$, Const ${ }^{+}$, and $\mathscr{C}$ are the families of all positive lower semicontinuous, functions all positive constant functions, and all continuous functions, respectively, defined on $\mathbb{R}$.

Another important result is due to Alinayat in [5]. We will state it as a theorem.

Theorem 2. If $f: \mathbb{R} \rightarrow \mathbb{R}$ is continuous, then for each $\epsilon>0$ there exists a positive continuous function $\delta: \mathbb{R} \rightarrow \mathbb{R}^{+}$such that for any $x, y \in \mathbb{R}$

$$
|x-y|<\max \{\delta(x), \delta(y)\} \Longrightarrow|f(x)-f(y)|<\epsilon .
$$

We are now ready to prove our main result. To make the result as general as possible we consider real-valued Baire-1 functions defined in the Euclidean space $X=\mathbb{R}^{n}$ for $n \geq 1$ with the standard Euclidean norm.

Theorem 3. The following statements are equivalent.

(1) $f: \mathbb{R} \rightarrow \mathbb{R}$ is continuous.

(2) For every Baire-1 function $g: \mathbb{R}^{n} \rightarrow \mathbb{R}, f \circ g: \mathbb{R}^{n} \rightarrow$ $\mathbb{R}$ is Baire-1.

That is, $f$ is a left Baire-1 compositor.

Proof. It is easy to see that if $f$ is continuous, then $f$ is a left Baire-1 compositor. Suppose $f$ is a left Baire- 1 compositor. If, on the contrary, we suppose $f$ has a discontinuity point $x_{0}$, we may assume without loss of generality that $x_{0}$ is the only point of discontinuity of $f$. Then $f$ may take one of the following forms:

(I)

$$
f(x)= \begin{cases}g_{1}(x), & x \neq x_{0} \\ L, & x=x_{0}\end{cases}
$$

with $f\left(x_{0}^{-}\right) \neq L$ or $f\left(x_{0}^{+}\right) \neq L$,
(II)

$$
f(x)= \begin{cases}g_{1}(x), & x \leq x_{0} ; \\ g_{2}(x), & x>x_{0}, \text { or }\end{cases}
$$

$$
f(x)= \begin{cases}g_{1}(x), & x<x_{0} \\ g_{2}(x), & x \geq x_{0} .\end{cases}
$$

Note that the functions $g_{1}$ and $g_{2}$ are continuous functions on their own domain. We will tackle Case I. Let $r_{1}, r_{2}, r_{3}, \ldots, r_{k}, \ldots$ be an enumeration of the cross product $\mathbb{Q} \times \mathbb{Q} \times \cdots \times \mathbb{Q}=\mathbb{Q}^{n} \subset \mathbb{R}^{n}$. Let $h: \mathbb{R}^{n} \rightarrow \mathbb{R}$ such that

$$
h(x)= \begin{cases}x_{0}+\frac{1}{k}, & x=r_{k} ; \\ x_{0}, & \text { otherwise. }\end{cases}
$$

First, we need to show that $h$ is Baire-1. We will adapt a wellknown technique in showing that $h$ is continuous on the complement $\left(\mathbb{Q}^{n}\right)^{c}$ of $\mathbb{Q}^{n}$. If $\xi \in \mathbb{Q}^{n}$, then $h$ is discontinuous at $\xi$. Let $\epsilon>0$ and $\xi$ be an arbitrary element of $\left(\mathbb{Q}^{n}\right)^{c}$. We can find a natural number $n$ such that $1 / n<\epsilon$. For such $n$, choose $\delta>0$ such that $\left\|\xi-r_{i}\right\| \geq \delta$ for each $1 \leq i \leq n$. Suppose $\|x-\xi\|<\delta$. Then either $x \in\left(\mathbb{Q}^{n}\right)^{c}$ or $x=r_{k}$ for some $k>n$. If $x \in\left(\mathbb{Q}^{n}\right)^{c}$, then clearly $|h(x)-h(\xi)|<\epsilon$. If $x=r_{k}$ for some $k>n$, then $|h(x)-h(\xi)|=\left|\left(x_{0}+(1 / k)\right)-x_{0}\right|=1 / k<1 / n<\epsilon$. Hence, $h$ is continuous on the complement of $\mathbb{Q}^{n}$. If $K$ is any nonempty perfect set in $\mathbb{R}^{n}$, then $K$ is uncountable. Hence, $\left.h\right|_{K}$ has at least a point of continuity in $K$. Consequently, $h$ is Baire-1. Next, we show that $f \circ h$ is not Baire- 1 so that $f$ is not a left Baire-1 compositor, a contradiction to our hypothesis. Now,

$$
(f \circ h)(x)= \begin{cases}g_{1}\left(x_{0}+\frac{1}{k}\right), & x=r_{k} \\ L, & \text { otherwise. }\end{cases}
$$

It is straightforward to show that $f \circ h$ is discontinuous everywhere in some set of the second category in $X$. Case II may be dealt with similarly with $h: \mathbb{R}^{n} \rightarrow \mathbb{R}$ given by

$$
h(x)= \begin{cases}x_{0}-\frac{1}{k}, & x=r_{k} ; \\ x_{0}, & \text { otherwise. }\end{cases}
$$

Following the same line of reasoning in Case I, one can show that $f \circ h$ is discontinuous everywhere in some set of the second category in $X$. The remaining case can be proved similarly. All these arguments show that $f \circ h$ is not Baire1. Therefore, $f$ must be a continuous function.

A part of the statement of the next result is contained in [2]. This inclusion is in Theorem 1 . With our main result, Theorem 1 (Theorem 11 in [2]) admits a very straightforward proof. 
Corollary 4. Let $\mathscr{C}^{+}$be the family of all positive continuous functions, Const ${ }^{+}$the family of all positive constant functions, $l s c^{+}$the family of all positive lower semicontinuous functions, and $\mathscr{C}$ the family of all continuous functions defined on $\mathbb{R}$. Then

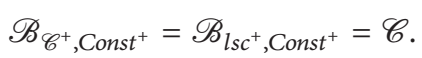

Proof. The inclusion $\mathscr{C} \subset \mathscr{B}_{\mathscr{C}^{+}, \text {Const }^{+}}$is immediate from Theorem 2. Let $f \in \mathscr{B}_{\mathscr{C}^{+} \text {,Const }}$. We will show that $f$ is a left Baire-1 compositor so that by Theorem $3 f$ is continuous. Let $g: \mathbb{R} \rightarrow \mathbb{R}$ be any Baire-1 function and let $\epsilon>0$. Then there is a positive continuous function $\delta: \mathbb{R} \rightarrow \mathbb{R}^{+}$such that for any $x, y \in \mathbb{R}$

$$
|x-y|<\delta(x) \wedge \delta(y) \Longrightarrow|f(x)-f(y)|<\epsilon .
$$

Since $g$ is Baire-1, there is a positive function $\lambda: \mathbb{R} \rightarrow \mathbb{R}^{+}$ such that for any $x, y \in \mathbb{R}$

$$
\begin{aligned}
|x-y|<\lambda(x) \wedge \lambda(y) \\
\quad \Longrightarrow|g(x)-g(y)|<\delta(g(x)) \wedge \delta(g(y)) .
\end{aligned}
$$

It follows that for any $x, y \in \mathbb{R}$

$$
|x-y|<\lambda(x) \wedge \lambda(y) \Longrightarrow|f(g(x))-f(g(y))|<\epsilon .
$$

Thus, $f \in \mathscr{C}$. Since $\mathscr{B}_{\mathscr{C}^{+}, \text {Const }}{ }^{+} \subset \mathscr{B}_{\text {lsc }^{+}, \text {Const }^{+}}$, then it follows that

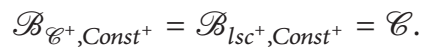

The proof is complete.

\section{Acknowledgments}

The authors are thankful to the referee for the helpful suggestions that led to the improvement of the current paper. Moreover, the first author wishes to thank the Department of Science and Technology through the Science Education Institute for the financial support while this research was ongoing.

\section{References}

[1] P.-Y. Lee, W.-K. Tang, and D. Zhao, "An equivalent definition of functions of the first Baire class," Proceedings of the American Mathematical Society, vol. 129, no. 8, pp. 2273-2275, 2001.

[2] A. Bakowska and R. J. Pawlak, "On some characterizations of Baire class one functions and Baire class one like functions," Tatra Mountains Mathematical Publications, vol. 46, pp. 91-106, 2010.

[3] J. P. Fenecios and E. A. Cabral, " $K$-continuous functions and right $B_{1}$ compositors," Journal of the Indonesian Mathematical Society, vol. 18, no. 1, pp. 37-44, 2012.

[4] D. Zhao, "Functions whose composition with Baire class one functions are Baire class one," Soochow Journal of Mathematics, vol. 33, no. 4, pp. 543-551, 2007.

[5] A. Alinayat, " $\delta$ as a continuous function of $x$ and $\varepsilon$," The American Mathematical Monthly, vol. 107, no. 2, pp. 151-155, 2000. 


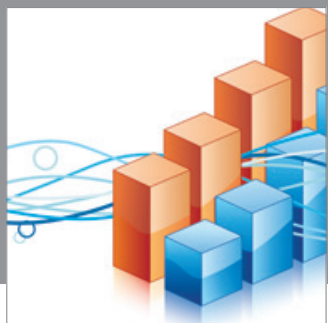

Advances in

Operations Research

mansans

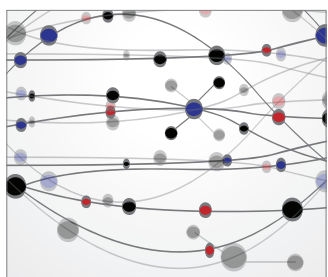

The Scientific World Journal
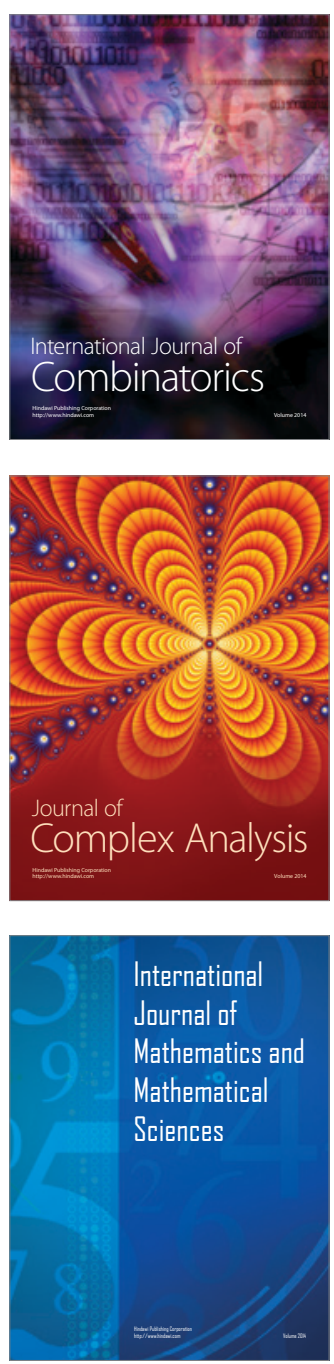
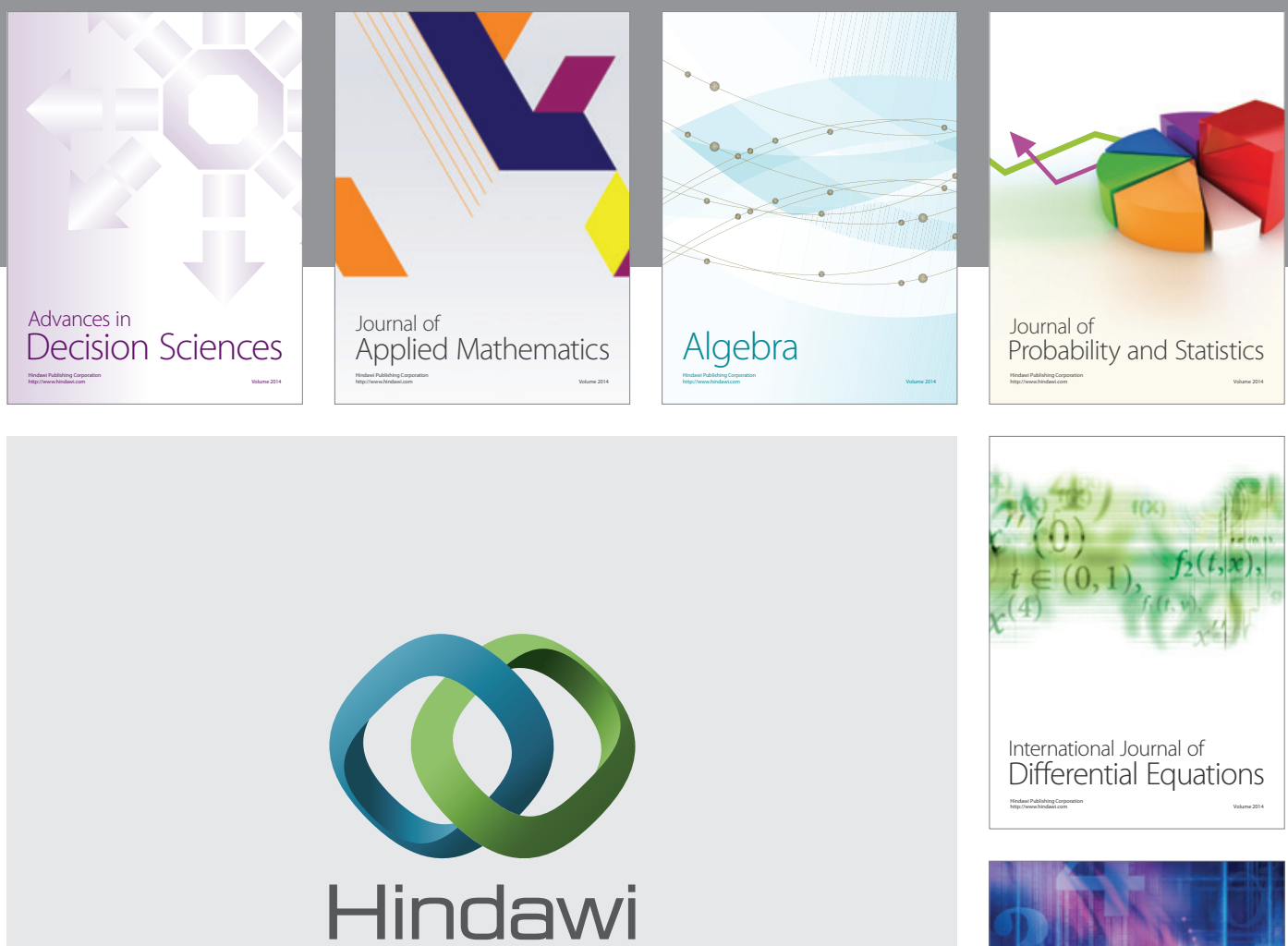

Submit your manuscripts at http://www.hindawi.com
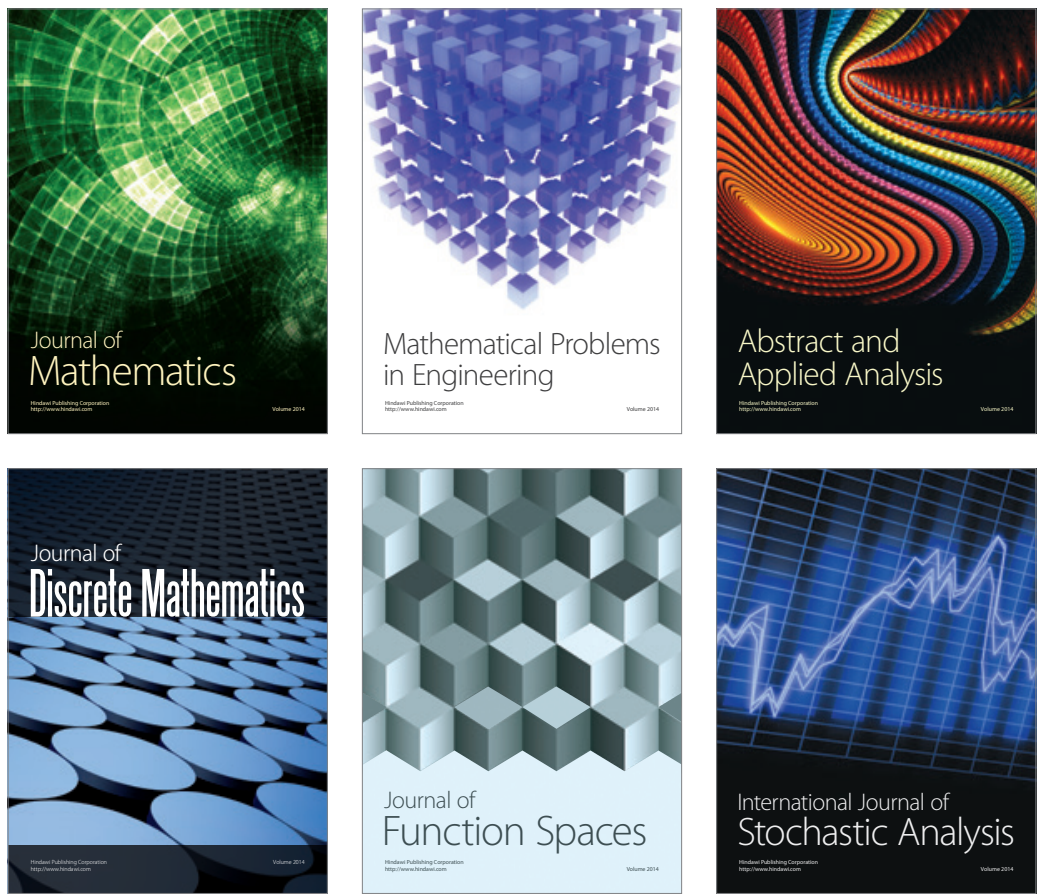

Journal of

Function Spaces

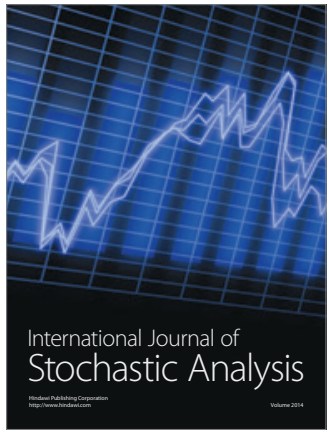

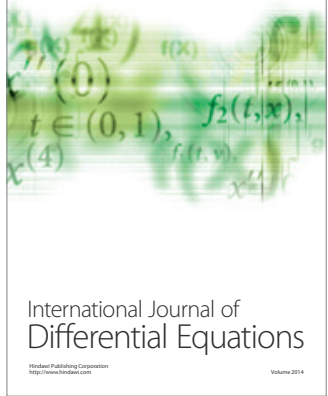
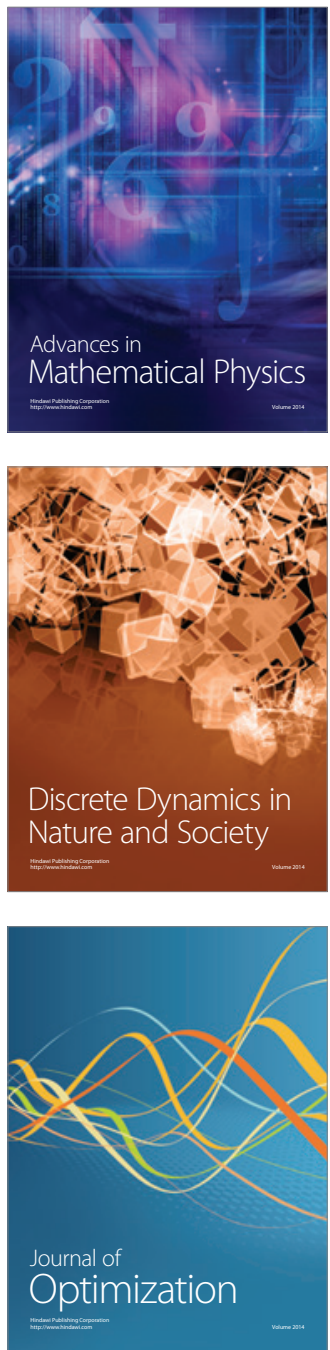